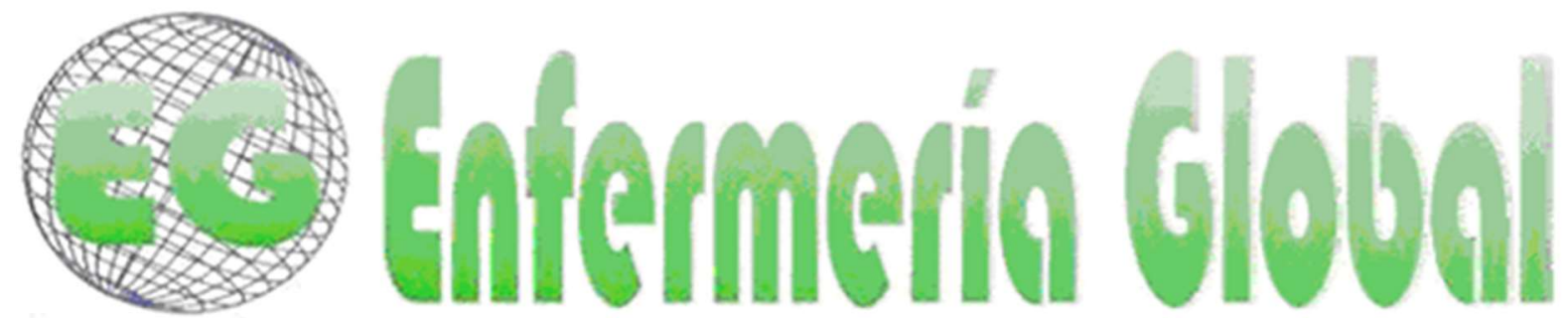

\title{
ORIGINALES
}

\section{Evaluación de la calidad de vida en Esclerosis Múltiple a través del MSQOL-54 y su relación con la salud de la persona}

Assessment of Quality of Life in Multiple Sclerosis through the MSQOL-54 and its relation to the health of the caregiver

\author{
M. Dolores Martínez-Espejo' \\ Rosa M. Limiñana-Gras ${ }^{1}$ \\ Rosa M. Patró-Hernández ${ }^{1}$, \\ José E. Meca Lallana ${ }^{2}$ \\ Encarnación Aznar Robles ${ }^{2}$ \\ M. Carmen Márquez Rebollo² \\ 1 Universidad de Murcia. España. liminana@um.es \\ ${ }^{2}$ Hospital Clínico Universitario Virgen de la Arrixaca. Murcia. España.
}

\section{https://doi.org/10.6018/eglobal.474161}

Recibido: 23/03/2021

Aceptado: 10/07/2021

\section{RESUMEN:}

Objetivo: El objetivo del presente estudio fue evaluar la Calidad de Vida Relacionada con la salud (CVRS) en personas con Esclerosis Múltiple (EM) mediante el cuestionario MSQOL-54 y analizar su relación con la salud de las personas cuidadoras principales a través del cuestionario GHQ-12 y un cuestionario de salud física autopercibida.

Metodología: Se evaluó a 115 personas con diagnóstico de EM y a 79 personas cuidadoras principales.

Resultados: Los resultados obtenidos señalan la significativa afectación de la CVRS de las personas con EM y su relación con la salud de las personas cuidadoras principales. Se encontraron relaciones significativas entre la salud física y mental de la persona con EM y un mayor número de enfermedades, peor salud mental y menor autopercepción de salud en la persona cuidadora.

Conclusiones: Los resultados obtenidos señalan la significativa afectación de la CVRS de las personas con EM y su relación directa con la salud de las personas cuidadoras principales.

Palabras clave: esclerosis múltiple, calidad de vida, calidad de vida relacionada con la salud, cuidadores, salud mental

\section{ABSTRACT:}

Objective: The aim of the present study was to evaluate the health-related quality of life (HRQLL) in patients with Multiple Sclerosis (MS) through the MSQOL-54 questionnaire and to analyse its relationship with the health of primary caregivers through the GHQ-12 questionnaire and a questionnaire on self-perceived physical health.

Materials and Methods: A total of 115 subjects diagnosed with MS and 79 primary caregivers were evaluated. 
Results: The results obtained show the significance of effects on HRQoL of patients with MS and its relationship to the health of primary caregivers. Significant relationships were found between the physical and mental health of the person with MS and an increased number of illnesses, poorer mental health and lower self-perception of health in the caregiver.

Conclusions: The results obtained point to the significant impairment of the HRQoL of people with MS, and its direct relationship with the health of primary caregivers.

Keywords: multiple sclerosis, quality of life, health-related quality of life, caregivers, mental health

\section{INTRODUCCIÓN}

La esclerosis múltiple (EM) es una enfermedad autoinmune clínicamente muy heterogénea, progresiva e impredecible que tiene un gran impacto sobre la calidad de vida de los pacientes y sus familiares ${ }^{(1)}$. Numerosos estudios coinciden en que afecta negativamente de forma más intensa que cualquier otra enfermedad crónica y con empeoramiento en al menos un tercio de los pacientes tras el diagnóstico ${ }^{(2)}$. Para una persona con EM, la calidad de vida es el resultado de la interacción de muchos factores, como son el tipo de enfermedad y su evolución, la etapa en la que se produce la enfermedad, la personalidad de la persona que lo sufre y su capacidad de adaptación a la enfermedad, la intensidad con la que afecta a su entorno y cambia su vida, o el apoyo social que perciba, entre otros ${ }^{(3)}$.

La aplicación del concepto de calidad de vida al ámbito de la salud conduce al término de "calidad de vida relacionada con la salud" (CVRS), englobando de forma más específica los dominios físicos, emocionales y sociales de la salud(4). En relación a la dimensión física, los principales factores que se han señalado como relevantes en la CVRS de la persona con EM son los que provocan una discapacidad funcional (alteraciones motoras o sensitivas), la fatiga, el dolor y los problemas sexuales o urinarios. En la dimensión emocional o psicológica se destacan factores como la ansiedad, la depresión, la pérdida de funciones cognitivas o la actitud frente a la

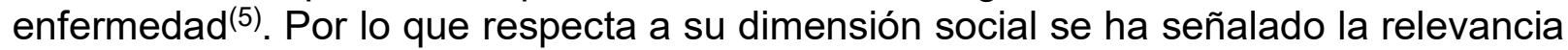
de la estigmatización que la patología produce en la red social del paciente, la pérdida de amistades y relaciones por falta de adaptación a la nueva situación y las repercusiones negativas que pueden darse en su vida laboral(6).

La medición de la CVRS se considera un ámbito de evaluación fundamental en pacientes con EM, ya que permite obtener una valoración sobre el impacto que la enfermedad tiene en la persona, permitiendo identificar las necesidades del paciente, pronosticar el impacto de la enfermedad en las dimensiones físicas y psicológicas de su calidad de vida $y$, a través de una valoración periódica, tener la posibilidad de identificar posibles cambios y optimizar el tratamiento (7). En los últimos años, diferentes estudios han señalado el impacto de la EM en la CVRS, no solo de los pacientes, sino también en sus cuidadores señalándose también la importancia del estudio de la relación entre estas dos medidas ${ }^{(8,9)}$.

La finalidad del presente estudio es obtener una medida adecuada de la CVRS de las personas con EM y de la salud de las personas cuidadoras principales y analizar la relación entre ambas, con el propósito de confirmar las principales repercusiones de esta enfermedad y aportar nuevas evidencias que sirvan para el desarrollo de intervenciones que sean eficaces en la prevención y mejora de la CVRS de la persona con EM y en la salud de las personas cuidadoras. Para ello, se plantean dos objetivos: a) evaluar la CVRS física y mental de las personas con EM a través de un 
instrumento de medida específico para la enfermedad y b) explorar la salud de los cuidadores o cuidadoras y su relación con la CVRS de las personas con EM.

\section{MÉTODO}

La prevalencia de la EM en la población murciana ha sido estimada en un 88 casos/100.000 habitantes ${ }^{(10)}$. La muestra de estudio se compuso de 115 personas con diagnóstico de EM, 30 de ellas procedentes a la Asociación de Esclerosis Múltiple de la Región de Murcia y 85 de la Unidad de Esclerosis Múltiple del Hospital Clínico Universitario Virgen de la Arrixaca de Murcia (HCUVA), incluyendo una muestra de 79 personas cuidadoras informales de la mencionada Unidad de Esclerosis Múltiple del HCUVA. El 70,4\% $(n=81)$ de las personas con diagnóstico de EM fueron mujeres y el $29,5 \%(n=34)$ hombres, siendo en el grupo de personas cuidadoras el $57 \% \quad(n=45)$ mujeres y el $43 \%(n=34)$ hombres. Los participantes fueron seleccionados utilizando un muestreo por selección intencionada o muestreo de conveniencia, a lo largo de 12 meses aproximadamente, desde enero de 2013 hasta enero de 2014.

Todos los participantes recibieron información comprensible y adecuada proporcionada por los responsables del proyecto, aprobado por la Comisión Ética de la Universidad de Murcia, participando de forma voluntaria y firmando un consentimiento informado.

Los participantes con EM cumplimentaron dos cuestionarios:

- Un breve Cuestionario sobre variables sociodemográficas y clínicas, en el que se recogieron datos relativos a la enfermedad y al grado de discapacidad (establecido según R.D. 1856/2009 de 4 de diciembre) completados posteriormente con los datos de sus historias clínicas.

- $\quad$ El cuestionario Múltiple Sclerosis Quality of Life- 54 (MSQOL-54) (11), en su versión española ${ }^{(12)}$, como medida multidimensional de la CVRS que combina elementos genéricos y específicos sobre la EM. Consta de 54 ítems de tipo Likert que evalúan intensidad o frecuencia referidos a las 4 últimas semanas. La puntuación total de cada una de las dimensiones abarca un rango de 0-100, donde un valor más elevado indica mejor CVRS. Se obtienen dos subtotales, mediante las puntuaciones de cada dimensión, correspondientes a dos escalas: Salud Mental y Salud Física. Las 12 subescalas del instrumento muestran una buena consistencia interna con alfas de Cronbach que van desde 0.75 a 0.96 . La confiabilidad testretest para estas 12 subescalas también es correcta, con coeficientes de correlación intraclase que varían de 0.66 a 0.96 . La consistencia interna en la muestra objeto de estudio obtuvo buenos valores $(\alpha=0.783)$.

Los cuidadores cumplimentaron en primer lugar un cuestionario sobre variables sociodemográficas y de cuidado, con una consistencia interna para nuestra muestra alta $(\alpha=0,87)$, y varias medidas de salud:

- El General Health Questionare-12 (GHQ-12)(13), instrumento de cribaje y detección de malestar psicológico que ha sido validado para la población adulta española por Sánchez-López y Dresch(14). Formado por 12 ítems tipo Likert de cuatro opciones de respuesta $(0-1-2-3)$, con rango de 0 a 36 puntos, se considera que 
puntuaciones por encima de 16 indicarían problemas psicológicos. La consistencia interna de esta medida en la población española es de $\alpha=0.76$, obteniendo en la muestra objeto de estudio un valor de $\alpha=0.89$.

- Un cuestionario de salud física autopercibida (escala Likert de 5 puntos, de $1=$ muy bueno a $5=$ muy malo), con una consistencia interna de $\alpha=0,87$ para nuestra muestra, y otro sobre número de problemas de salud (28 dolencias o enfermedades), ambos basados en los indicadores de salud de la Encuesta Nacional de Salud(15).

Se siguieron dos procedimientos para la recogida de datos. En un primer momento se hizo en la Asociación de Esclerosis Múltiple de la Región de Murcia, a través del correo ordinario, un proceso lento en el que de los 60 cuestionarios repartidos se recogieron 30. Posteriormente, la recogida de datos se llevó a cabo en la Unidad de Esclerosis Múltiple del Hospital Clínico Universitario Virgen de la Arrixaca de Murcia, unidad de referencia regional y nacional que cuenta con una media aproximada de 700 pacientes atendidos anualmente; el procedimiento fue la administración directa de los cuestionarios a los participantes voluntarios, gracias a la colaboración de los/las profesionales de la Unidad.

Se realizó un diseño descriptivo y correlacional, utilizándose el paquete estadístico SPSS en su versión 22. Se utilizaron estadísticos descriptivos desagregando los mismos en función de la variable sexo dada la relevancia de tener en cuenta la perspectiva de género en las investigaciones llevadas a cabo en contextos de salud ${ }^{(16)}$. Se utilizó el estadístico $t$ y el estadístico $F$ para la comparación de medias en función del sexo, edad, nivel educativo, situación laboral y estado civil, y el estadístico $r$ de Pearson para hallar el sentido y la significación de la relación entre variables de CVRS de las personas con EM y variables clínicas y relativas a la salud de las personas cuidadoras.

\section{RESULTADOS}

En la tabla 1 se muestran los estadísticos descriptivos totales y desagregados por sexo para las variables demográficas en los 115 pacientes con esclerosis múltiple y las 79 personas cuidadoras principales.

Tabla 1. Estadísticos descriptivos de las personas con E.M. y las personas cuidadoras principales

\begin{tabular}{|c|c|c|c|c|c|c|c|}
\hline \multirow[b]{2}{*}{ Variables } & \multicolumn{3}{|c|}{ Personas con EM } & \multirow[b]{2}{*}{ Variables } & \multicolumn{3}{|c|}{ Personas cuidadoras } \\
\hline & $\begin{array}{l}\text { Mujeres } \\
(n=81)\end{array}$ & $\begin{array}{c}\text { Hombres } \\
(\mathrm{n}=34)\end{array}$ & $\begin{array}{c}\text { Total } \\
(\mathrm{N}=115)\end{array}$ & & $\begin{array}{c}\text { Mujeres } \\
(n=45)\end{array}$ & $\begin{array}{l}\text { Hombres } \\
(n=34)\end{array}$ & $\begin{array}{c}\text { Total } \\
(\mathrm{N}=79)\end{array}$ \\
\hline Edad (años) & & & & Edad (años) & & & \\
\hline $20-30$ & $19(23.8 \%)$ & $6(17.6 \%)$ & $25(21.9 \%)$ & $20-30$ & $12(26.7 \%)$ & $2(5.9 \%)$ & $14(17.7 \%)$ \\
\hline $31-40$ & $27(33.8 \%)$ & $11(32.4 \%)$ & $38(33.3 \%)$ & $31-40$ & $8(17.8 \%)$ & $9(26.5 \%)$ & $17(21.5 \%)$ \\
\hline $41-50$ & $23(28.8 \%)$ & $11(32.4 \%)$ & $34(29.8 \%)$ & $41-50$ & $13(28.9 \%)$ & $13(38.2 \%)$ & $26(32.9 \%)$ \\
\hline $51-65$ & $11(13.8 \%)$ & $6(17.6 \%)$ & $17(14.9 \%)$ & $51-65$ & $10(22.2 \%)$ & $8(23.5 \%)$ & $18(22.8 \%)$ \\
\hline & & & & $66-75$ & $2(4.4 \%)$ & $2(5.9 \%)$ & $4(5.1 \%)$ \\
\hline
\end{tabular}




\begin{tabular}{|c|c|c|c|c|c|c|c|}
\hline \multicolumn{5}{|c|}{ Personas con EM } & \multicolumn{3}{|c|}{ Personas cuidadoras } \\
\hline Variables & $\begin{array}{l}\text { Mujeres } \\
(n=81)\end{array}$ & $\begin{array}{c}\text { Hombres } \\
(n=34)\end{array}$ & $\begin{array}{c}\text { Total } \\
(\mathrm{N}=115)\end{array}$ & Variables & $\begin{array}{l}\text { Mujeres } \\
(n=45)\end{array}$ & $\begin{array}{l}\text { Hombres } \\
(n=34)\end{array}$ & $\begin{array}{c}\text { Total } \\
(\mathrm{N}=79)\end{array}$ \\
\hline $\begin{array}{l}\text { N. educativo } \\
\text { Primarios }\end{array}$ & & & & $\begin{array}{l}\text { N. educativo } \\
\text { Primarios }\end{array}$ & & & \\
\hline Secundarios & $28(34.6 \%)$ & $12(35.3 \%)$ & $40(34.8 \%)$ & Secundarios & $15(33.3 \%)$ & $14(42.4 \%)$ & $29(37.2 \%)$ \\
\hline F.P. & $12(14.8 \%)$ & $6(17.6 \%)$ & $18(15.7 \%)$ & F. P. & $8(17.8 \%)$ & $5(15.2 \%)$ & $13(16.7 \%)$ \\
\hline Superiores & $16(19.8 \%)$ & $7(20.6 \%)$ & $23(20.0 \%)$ & Superiores & $6(13.3 \%)$ & $7(21.2 \%)$ & $13(16.7 \%)$ \\
\hline No reglada & $\begin{array}{r}23(28.4 \%) \\
2(2.5 \%)\end{array}$ & $\begin{array}{r}9(26.5 \%) \\
0(0.0 \%)\end{array}$ & $\begin{array}{r}32(27.8 \%) \\
2(1.7 \%)\end{array}$ & No reglada & $\begin{array}{r}14(31.1 \%) \\
2(4.4 \%)\end{array}$ & $\begin{array}{r}7(21.2 \%) \\
0(0.0 \%)\end{array}$ & $\begin{array}{r}21(26.9 \%) \\
2(2.6 \%)\end{array}$ \\
\hline Sit. laboral & & & & Sit. laboral & & & \\
\hline Trabaja & $29(36.3 \%)$ & $15(44.1 \%)$ & $44(38.6 \%)$ & Trabaja & $22(48.9 \%)$ & $27(79.4 \%)$ & $49(62.0 \%)$ \\
\hline No trabaja & $49(61.3 \%)$ & $19(55.9 \%)$ & $68(59.6 \%)$ & No trabaja & $21(46.7 \%)$ & $7(20.6 \%)$ & $28(35.4 \%)$ \\
\hline Act no rem & $2(2.5 \%)$ & $0(0.0 \%)$ & $2(1.8 \%)$ & Act no rem & $2(4.4 \%)$ & $0(0.0 \%)$ & $2(2.6 \%)$ \\
\hline Estado civil & & & & Estado civil & & & \\
\hline Soltero/a & $27(33.8 \%)$ & $4(11.8 \%)$ & $31(27.2 \%)$ & Soltero/a & $9(20.0 \%)$ & $6(17.6 \%)$ & $15(19.0 \%)$ \\
\hline Casado/a & $48(60.0 \%)$ & $27(79.4 \%)$ & $75(65.8 \%)$ & Casado/a & $31(68.9 \%)$ & $27(79.4 \%)$ & $58(73.4 \%)$ \\
\hline Separado/a & $5(6.3 \%)$ & $3(8.8 \%)$ & $8(7.0 \%)$ & Separado/a & $5(11.1 \%)$ & $1(2.9 \%)$ & $6(7.6 \%)$ \\
\hline $\begin{array}{l}\text { Tiempo de } \\
\text { enfermedad a }\end{array}$ & $3.86 \pm 1.11$ & $3.96 \pm 1.19$ & $3.89 \pm 1.13$ & $\begin{array}{l}\text { Tiempo } \\
\text { como } \\
\text { cuidador a }\end{array}$ & $3.86 \pm 1.32$ & $4.12 \pm 1.22$ & $3.97 \pm 1.28$ \\
\hline $\begin{array}{l}\text { Grado de } \\
\text { discapacidada }^{a}\end{array}$ & $3.14 \pm 1.48$ & $3.38 \pm 1.51$ & $3.19 \pm 1.47$ & $\begin{array}{l}\text { Relación con } \\
\text { paciente }\end{array}$ & & & \\
\hline & & & & Padre/Madre & $13(28.9 \%)$ & $3(8.8 \%)$ & $16(20,3 \%)$ \\
\hline & & & & Hijo/a & $4(8.9 \%)$ & $1(2.9 \%)$ & $5(6.3 \%)$ \\
\hline & & & & Esposo/a & $20(36.4 \%)$ & $24(43.6 \%)$ & $24(30.4 \%)$ \\
\hline & & & & Otros & $8(17.8 \%)$ & $6(17.6 \%)$ & $14(17.7 \%)$ \\
\hline
\end{tabular}

${ }^{\mathrm{a}}$ media \pm desviación típica del tiempo en años

La tabla 2 presenta los estadísticos descriptivos para las puntuaciones de las subescalas que conforman el MSQOL-54 y los resultados del contraste de medias en función del sexo. Los resultados en Salud Física Total, con una puntuación total media de 53.40 sobre 100 , indican que los participantes de nuestra muestra tienen una CVRS Física moderada y que la subescala con menores puntuaciones es la relativa a las Limitaciones físicas que les produce la enfermedad. Respecto a la CVRS Mental, con una puntuación total media de 57.18, destaca la mayor afectación de la subescala relacionada con la Función cognitiva. No se encontraron diferencias significativas entre hombres y mujeres, ni en la puntuación total de las escalas Salud Física y Salud Mental ni en ninguna de las subescalas ( $p>.05)$.

Tabla 2. Puntuaciones medias del Multiple Sclerosis Quality of Life 54 (MSQOL-54) y comparación en función del sexo

\begin{tabular}{lrllr}
\hline MSQOL-54 & $\begin{array}{l}\text { Total } \\
(\mathbf{n = 1 1 5 )} \\
\text { Media (DT) }\end{array}$ & $\begin{array}{l}\text { Mujeres } \\
(\mathbf{n = 8 1 )} \\
\text { Media(DT) }\end{array}$ & $\begin{array}{l}\text { Hombres } \\
(\mathbf{n = 3 4 )} \\
\text { Media(DT) }\end{array}$ & \multicolumn{1}{c}{$\mathbf{t}$} \\
\hline Salud Física Total & & & & \\
$\quad$ Salud física & $53.4(21.4)$ & $53.2(21.9)$ & $53.6(20.6)$ & .562 \\
Percepción salud & $9.2(5.6)$ & $9.4(5.6)$ & $8.8(5.4)$ & .721 \\
Energía & $7.5(3.4)$ & $7.6(3.4)$ & $7.1(3.5)$ & -.038 \\
Limitaciones físicas & $5.7(2.4)$ & $5.7(2.4)$ & $5.7(2.5)$ & -.046 \\
\hline
\end{tabular}




\begin{tabular}{|c|c|c|c|c|}
\hline MSQOL-54 & $\begin{array}{l}\text { Total } \\
(\mathrm{n}=115) \\
\text { Media (DT) }\end{array}$ & $\begin{array}{l}\text { Mujeres } \\
(\mathrm{n}=81) \\
\text { Media(DT) }\end{array}$ & $\begin{array}{l}\text { Hombres } \\
(n=34) \\
\text { Media(DT) }\end{array}$ & $\mathbf{t}$ \\
\hline Dolor & $7.4(2.8)$ & $7.1(2.8)$ & $8.1(2.6)$ & -1.244 \\
\hline Función sexual & $5.2(2.5)$ & $5.0(2.6)$ & $5.7(2.3)$ & .364 \\
\hline Función social & $8.2(2.9)$ & $8.3(2.8)$ & $8.1(3.0)$ & .769 \\
\hline Preocupación salud & $6.7(3.0)$ & $6.9(3.0)$ & $6.4(2.9)$ & -.081 \\
\hline Salud Mental Total & $57.1(21.0)$ & $56.2(21.2)$ & $59.4(20.7)$ & -.76 \\
\hline Calidad vida general & $10.8(3.2)$ & $10.9(3.2)$ & $10.6(3.3)$ & .45 \\
\hline Bienestar emocional & $17.2(4.8)$ & $16.9(4.9)$ & $18.0(4.7)$ & -1.03 \\
\hline Limitaciones problemas & & & & \\
\hline emocionales & $11.3(11.3)$ & $10.5(11.2)$ & $13.1(11.4)$ & -1.13 \\
\hline Función cognitiva & $9.11(3.9)$ & $8.9(3.8)$ & $9.5(4.2)$ & -.69 \\
\hline
\end{tabular}

La edad fue un factor relevante que se relaciona de forma positiva principalmente a la CVRS Física (ver tabla 3). En Salud Física Total, los análisis post hoc revelaron que las diferencias en esta variable se dieron entre el rango más joven, de 20 a 30 años con los rangos de más edad, de 41 a $50(p=.024)$ y de 51 a $65(p=.000)$. Como se observa el rango más joven fue el que mostró una media superior en Salud Física, aumentando la diferencia con el resto de grupos en la medida que aumentaba la edad. La media en el grupo de 31 a 40 también fue significativamente más elevada que la del grupo de 51 a $65(p=.020)$. La subescala de Salud Física también presentó resultados significativos en función de la edad, las diferencias se dieron entre el rango de 20 a 30 años con los rangos de 31 a $40(p=.001)$, de 41 a $50(p=.000)$ y de 51 a $65(p=.000)$. Como se observa el rango más joven fue el que mostró una media superior en la subescala, aumentando la diferencia con el resto de grupos en la medida que aumentaba la edad. Las medias en los grupos de 31 a 40 y de 41 a 50 también fueron significativamente superiores a la del grupo de 51 a $65(p=.000$ y $p=$ .041 respectivamente), pero no se diferenciaron entre sí. En limitaciones físicas también se obtuvieron diferencias significativas $(p<.05)$ en función de la edad de las personas con EM. Las diferencias se dieron entre el rango de 20 a 30 años con los rangos de 41 a $50(p=.014)$ y de 51 a $65(p=.001)$, siendo el grupo más joven el que mostró una calidad de vida superior en relación a las limitaciones físicas.

Otra de las variables que mostró diferencias significativas $(p<.05)$ en función de la edad fue el grado de dolor. Los resultados de las comparaciones post hoc arrojaron diferencias entre el rango de 51 a 65 años con el de 41 a 30 años $(p=.033)$ y el de 20 a 30 años $(p=.013)$, a partir de los 41 años no se hallaron diferencias significativas en la calidad de vida, en relación al grado de dolor. Los grupos más jóvenes mostraron una calidad de vida superior en esta variable. La Función sexual fue la última subescala de la Salud Física en la que se hallaron diferencias significativas según el grupo de edad $(p<.05)$. En las comparaciones post hoc el grupo de 51 a 65 se diferenció significativamente con todos los demás rangos de edad: de 20 a 30 años $(p=.003)$ de 31 a $40(p=.023)$, y de 41 a $50(p=.012)$, que no difirieron significativamente entre sí. Por lo tanto, se observa que la calidad de vida en relación con la función sexual se va reduciendo con la edad aunque esta disminución no es significativa hasta los 51 años.

Dentro de las subescalas relacionadas con Salud Mental solo se obtuvieron resultados marginalmente significativos en la de Calidad de vida general, entre el grupo de 20 a 30 años y el de 51 a 65 años ( $p=.055)$. 
Tabla 3. Resultados de los ANOVAs para las escalas y subescalas del MSQOL-54 en función de la edad

\begin{tabular}{|c|c|c|c|c|c|c|c|c|c|}
\hline \multirow{2}{*}{ MSQOL-54 } & \multicolumn{2}{|c|}{$\begin{array}{l}20-30 \text { años } \\
(\mathrm{n}=\mathbf{2 5})\end{array}$} & \multicolumn{2}{|c|}{$\begin{array}{c}31-40 \text { años } \\
(n=38)\end{array}$} & \multicolumn{2}{|c|}{$\begin{array}{c}41-50 \text { años } \\
(n=34)\end{array}$} & \multicolumn{2}{|c|}{$\begin{array}{c}51-65 \text { años } \\
(n=17)\end{array}$} & \multirow[t]{2}{*}{$\boldsymbol{F}$} \\
\hline & $M$ & $D T$ & $M$ & $D T$ & $M$ & $D T$ & $M$ & $D T$ & \\
\hline Salud Física Total & 65.11 & 22.20 & 55.09 & 21.33 & 49.92 & 18.04 & 37.90 & 16.61 & $6.72^{*}$ \\
\hline Salud Física & 14.09 & 2.96 & 10.11 & 5.11 & 7.45 & 4.98 & 3.65 & 4.21 & $2.26^{*}$ \\
\hline $\begin{array}{l}\text { Percepción de } \\
\text { Salud }\end{array}$ & 8.70 & 4.05 & 7.72 & 3.03 & 6.67 & 3.18 & 6.55 & 3.19 & 2.52 \\
\hline Energía & 6.82 & 1.95 & 5.35 & 2.64 & 5.51 & 2.50 & 5.05 & 2.36 & 5.53 \\
\hline Limitaciones físicas & 6.48 & 5.32 & 3.79 & 5.34 & 2.29 & 4.31 & 1.05 & 2.99 & $3.55^{*}$ \\
\hline Dolor & 8.19 & 2.47 & 7.74 & 2.49 & 7.32 & 3.12 & 5.53 & 2.71 & $4.74^{*}$ \\
\hline Función sexual & 6.05 & 1.88 & 5.35 & 2.75 & 5.54 & 2.30 & 3.28 & 2.75 & $1.91^{*}$ \\
\hline Función social & 8.90 & 2.33 & 8.16 & 3.12 & 8.50 & 2.58 & 6.82 & 3.54 & .70 \\
\hline $\begin{array}{l}\text { Preocupación } \\
\text { salud }\end{array}$ & 7.28 & 3.41 & 6.86 & 2.98 & 6.56 & 2.41 & 5.95 & 3.68 & 2.26 \\
\hline $\begin{array}{l}\text { Salud Mental } \\
\text { Total }\end{array}$ & 62.38 & 21.67 & 57.74 & 22.64 & 55.48 & 18.85 & 51.36 & 21.20 & 1.01 \\
\hline $\begin{array}{l}\text { Calidad de vida en } \\
\text { general }\end{array}$ & 12.22 & 3.29 & 10.71 & 3.32 & 10.39 & 2.82 & 9.66 & 3.22 & $2.60^{a}$ \\
\hline $\begin{array}{l}\text { Bienestar } \\
\text { emocional }\end{array}$ & 16.47 & 5.19 & 17.15 & 4.93 & 17.80 & 4.57 & 17.26 & 4.83 & .36 \\
\hline $\begin{array}{l}\text { Limitaciones probl } \\
\text { emocionales }\end{array}$ & 14.40 & 10.83 & 12.63 & 11.40 & 9.41 & 11.39 & 8.47 & 11.12 & 1.48 \\
\hline Función cognitiva & 10.02 & 3.52 & 8.50 & 4.52 & 9.52 & 3.52 & 8.38 & 4.03 & 1.05 \\
\hline
\end{tabular}

${ }^{*} p<.05 ;{ }^{a}$ marginalmente significativo $p=.055$

La CVRS presentó también diferencias significativas en función del nivel educativo (ver tabla 4). En Salud Física los que cursaron estudios superiores presentaron mejores puntuaciones que los que tenían estudios primarios $(p=.000)$. En Salud Mental los análisis post hoc revelaron que las diferencias en esta variable tan solo se dieron entre los que cursaron estudios superiores y todos los demás niveles, estudios primarios $(p=.000)$, estudios secundarios $(p=.029)$ y formación profesional $(p=$ $.012)$.

Tabla 42. Resultados de los ANOVAs para las escalas y subescalas del MSQOL-54 en función del nivel educativo

\begin{tabular}{llllll}
\hline & $\begin{array}{l}\text { Estudios } \\
\text { primarios } \\
(\mathbf{n = 4 0 )}\end{array}$ & $\begin{array}{l}\text { Estudios } \\
\text { secundarios } \\
(\mathbf{n = 1 8})\end{array}$ & $\begin{array}{l}\text { Formación } \\
\text { profesional } \\
(\mathbf{n = 2 3 )}\end{array}$ & $\begin{array}{l}\text { Estudios } \\
\text { superiores } \\
(\mathbf{n = 3 2})\end{array}$ & \\
MSQOL-54 & Media (DT) & Media (DT) & Media (DT) & Media (DT) & T \\
\hline Salud Física & $43.30(18.11)$ & $52.89(22,8)$ & $53.18(19.70)$ & $65.38(20.06)$ & $7.31^{*}$ \\
$\begin{array}{l}\text { Total } \\
\quad \text { Salud física }\end{array}$ & $7.38(5.10)$ & $9.77(5.44)$ & $8.76(5.75)$ & $11.39(5.55)$ & $3.36^{*}$ \\
$\quad$ Percepción & $6.01(3.43)$ & $7.31(3.97)$ & $8.54(3.34)$ & $8.63(2.92)$ & $4.82^{*}$ \\
\hline
\end{tabular}




\begin{tabular}{|c|c|c|c|c|c|}
\hline MSQOL-54 & 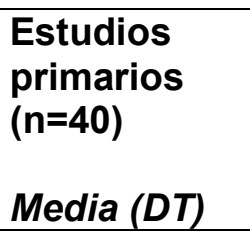 & $\begin{array}{l}\text { Estudios } \\
\text { secundarios } \\
(\mathrm{n}=18) \\
\text { Media }(D T)\end{array}$ & $\begin{array}{l}\text { Formación } \\
\text { profesional } \\
(\mathrm{n}=23) \\
\text { Media }(D T)\end{array}$ & 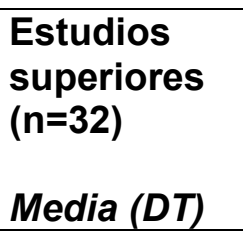 & \\
\hline \multicolumn{6}{|l|}{ salud } \\
\hline Energía & $4.57(2.33)$ & $6.10(2.90)$ & $5.86(2.01)$ & $6.71(2.30)$ & $5.22^{*}$ \\
\hline $\begin{array}{l}\text { Limitaciones } \\
\text { físicas }\end{array}$ & $1.57(3.52)$ & $2.83(4.65)$ & $3.26(5.18)$ & $6.28(5.51)$ & $6.15^{*}$ \\
\hline Dolor & $6.64(2.83)$ & $6.79(2.98)$ & $7.24(2.86)$ & $8.82(2.19)$ & $4.33^{*}$ \\
\hline Función sexual & $4.65(2.67)$ & $5.55(2.17)$ & $4.78(2.96)$ & $6.06(2.15)$ & 2.17 \\
\hline Función social & $8.05(3.06)$ & 7.63(3.11) & 7.69(3.12) & $9.25(2.39)$ & 1.86 \\
\hline $\begin{array}{l}\text { Preocupación } \\
\text { salud }\end{array}$ & $5.39(2.79)$ & $6.82(3.65)$ & 7.03(3.11) & $8.16(2.19)$ & $5.68^{*}$ \\
\hline $\begin{array}{l}\text { Salud Mental } \\
\text { Total }\end{array}$ & $50.67(19.49)$ & $51.67(22.3)$ & $54.62(21.62)$ & $69.76(16.62)$ & $6.42^{*}$ \\
\hline $\begin{array}{l}\text { Calidad de vida } \\
\text { general }\end{array}$ & $9.63(3.35)$ & $10.81(3.21)$ & $11.07(2.77)$ & $11.94(2.92)$ & $3.38^{*}$ \\
\hline $\begin{array}{l}\text { Bienestar } \\
\text { Emocional }\end{array}$ & $16.58(4.59)$ & $17.53(5.66)$ & $16.59(5.56)$ & $18.56(4.26)$ & 1.15 \\
\hline $\begin{array}{l}\text { Limitaciones } \\
\text { probl. emoc. }\end{array}$ & $9.60(11.04)$ & 7.11(10.2) & $9.04(11.64)$ & $17.50(9.83)$ & $5.16^{*}$ \\
\hline $\begin{array}{l}\text { Función } \\
\text { cognitiva }\end{array}$ & 7.98(3.68) & $7.54(4.42)$ & 8.96(4.04) & $11.36(3.03)$ & $6.21^{*}$ \\
\hline
\end{tabular}

La situación laboral mostró una relación significativa en todas las medidas de resultado, indicando que los pacientes que trabajan tienen mejor CVRS en la dimensión de salud física y mental que los que no trabajan (tabla 5).

Tabla 5. Resultados de la comparación de medias de las escalas y subescalas del MSQOL-54 en función de la situación laboral

\begin{tabular}{llll}
\hline & $\begin{array}{l}\text { Trabaja (n=44) } \\
\text { Media (DT) }\end{array}$ & $\begin{array}{l}\text { No trabaja (n=68) } \\
\text { Media (DT) }\end{array}$ & $\boldsymbol{t}$ \\
\hline MSQOL-54 & $66.54(20.31)$ & $44.26(17.18)$ & $6.23^{\star * *}$ \\
Salud Física Total & $12.86(3.90)$ & $6.80(5.30)$ & $6.95^{\star * *}$ \\
Salud física & $9.16(3.33)$ & $6.34(3.02)$ & $4.61^{* * *}$ \\
Percepción salud & $6.82(2.21)$ & $4.86(2.30)$ & $4.46^{\star * *}$ \\
Energía & $6.40(5.39)$ & $1.58(3.68)$ & $5.19^{\star * *}$ \\
Limitaciones físicas & $8.63(2.18)$ & $6.55(2.87)$ & $4.34^{* * *}$ \\
Dolor & $6.02(2.20)$ & $4.73(2.67)$ & $2.77^{* *}$ \\
Función sexual & $9.26(2.53)$ & $7.52(2.96)$ & 3.18 \\
Función social & $8.11(2.36)$ & $5.82(3.04)$ & $4.47^{\star * *}$ \\
Preocupación salud & $68.35(17.62)$ & $49.65(19.87)$ & $5.07^{\star * *}$ \\
Salud Mental Total & $12.40(2.66)$ & $9.75(3.20)$ & $4.55^{\star * *}$ \\
Calidad de vida general & $18.29(4.74)$ & $16.37(4.73)$ & $2.09^{*}$ \\
Bienestar emocional & & &
\end{tabular}




\begin{tabular}{llll}
\hline & $\begin{array}{l}\text { Trabaja }(\mathbf{n}=44) \\
\text { Media }(\mathbf{D T})\end{array}$ & $\begin{array}{l}\text { No trabaja }(\mathbf{n}=68) \\
\text { Media (DT) }\end{array}$ & $\boldsymbol{t}$ \\
\hline $\begin{array}{l}\text { Limitaciones probl. } \\
\text { emocionales }\end{array}$ & $17.09(10.29)$ & $7.76(10.43)$ & $4.64^{* * *}$ \\
$\begin{array}{l}\text { Función cognitiva } \\
{ }^{*} p<.05^{* *} p<01^{* * *} p<.001\end{array}$ & $10.22(3.60)$ & $8.34(4.01)$ & $2.51^{*}$ \\
\hline
\end{tabular}
${ }^{*} p<.05,{ }^{* *} p<.01,{ }^{* * *} p<.001$

Por último, también se encontraron diferencias en CVRS Física y Mental en función del estado civil (ver tabla 6$)$. La Salud Física Total $\left(F_{111}=6.54, p<.50\right)$ y Mental Total $\left(F_{111}=8.56, p<.05\right)$ fue superior en los solteros, seguido de los casados y la menor para los separados. Los análisis post hoc revelaron que las diferencias en Salud Física se dieron entre los solteros y casados $(p=.029)$, los solteros y separados $(p=$ $.000)$, y los casados y los separados ( $p=.023)$; y en Salud Mental entre los separados y solteros $(p=.000)$, y entre solteros y casados (Media $=56.74, p=.000)$.

Para la Salud Física Total los análisis post hoc revelaron que las diferencias en esta variable se dieron entre los tres grupos, los solteros con los casados $(p=.029)$ y separados $(p=.000)$, diferenciándose ambos grupos también entre sí $(p=.023)$; la media de Salud Física fue superior en los solteros, seguido de los casados y la menor para los separados. Como se puede apreciar en la tabla 6, para todas las subescalas se hallaron diferencias significativas en función del estado civil de los pacientes. Para las subescalas de Salud Física y de Dolor, los solteros difirieron significativamente en los resultados post hoc con los casados ( $p=.000$ y $p=.005$ respectivamente) y los separados ( $p=.004$ y $p=.015$ respectivamente), que no difirieron entre sí, siendo la calidad de vida en relación a estas dos subescalas superior en los solteros. En Percepción de la Salud y en Limitaciones físicas solo se encontraron diferencias significativas entre solteros y separados $(p=.029$ y $p=.022$ respectivamente), siendo la calidad de vida en relación a estas subescalas superior en los solteros. En energía/fatiga no se observaron diferencias entre solteros y casados, si entre estos dos y los separados $(p<.000$ y $p<.008)$, siendo los resultados superiores en los solteros.

Para la Salud Mental Total, las diferencias post hoc se dieron entre los separados en comparación con los grupos de solteros $(p=.000)$ y casados $(p=.000)$. Para la subescala de Calidad de Vida, sin embargo, las diferencias se dieron entre los tres grupos, los solteros con casados $(p=.009)$ y separados $(p=.000)$, y casados y solteros entre sí $(p=.004)$, siendo la media superior en los solteros, seguido de los casados y la menor para los separados. Para la subescala de Limitaciones por Problemas Emocionales, volvemos a encontrar las diferencias significativas en el análisis post hoc, entre separados con solteros $(p=.000)$ y casados $(p=.000)$, no difiriendo estos últimos entre sí; al igual que en la subescala de Función cognitiva, mostrando solo diferencias entre los separados con solteros $(p=.001)$ y casados $(p=$ .003), no difiriendo estos últimos entre sí. Estos resultados hay que interpretarlos con cautela dado el bajo $n$ en el grupo de separados. 
Tabla 6. Resultados de los ANOVAs para las escalas y subescalas del MSQOL-54 en función del estado civil.

\begin{tabular}{|c|c|c|c|c|c|c|c|}
\hline \multirow{2}{*}{$\begin{array}{l}\text { Multiple Sclerosis } \\
\text { Quality of Life } 54 \\
\text { (MSQOL-54) }\end{array}$} & \multicolumn{2}{|c|}{$\begin{array}{l}\text { Soltero/a } \\
(n=31)\end{array}$} & \multicolumn{2}{|c|}{$\begin{array}{l}\text { Casado/a } \\
(n=75)\end{array}$} & \multicolumn{2}{|c|}{$\begin{array}{l}\text { Separado/a } \\
(n=8)\end{array}$} & \multirow[b]{2}{*}{$\boldsymbol{F}$} \\
\hline & $M$ & $D T$ & $M$ & $D T$ & $M$ & $D T$ & \\
\hline Salud Física Total & 63.14 & 20.76 & 51.25 & 21.14 & 36.68 & 11.22 & $6.54^{* *}$ \\
\hline Salud Física & 12.69 & 4.89 & 8.07 & 5.51 & 6.90 & 3.38 & $9.38^{* * *}$ \\
\hline Percepción de Salud & 8.66 & 3.25 & 7.26 & 3.47 & 5.20 & 2.50 & $3.90^{*}$ \\
\hline Energía & 6.73 & 2.27 & 5.61 & 2.36 & 2.94 & 2.43 & $8.58^{* * *}$ \\
\hline Limitaciones físicas & 5.22 & 5.42 & 3.20 & 4.91 & .00 & .00 & $4.10^{*}$ \\
\hline Dolor & 8.73 & 2.02 & 7.11 & 2.95 & 5.42 & 2.41 & $6.35^{* *}$ \\
\hline Función sexual & 5.93 & 1.97 & 5.04 & 2.68 & 4.77 & 3.18 & 1.47 \\
\hline Función social & 8.77 & 2.61 & 8.28 & 3.00 & 6.12 & 2.69 & 2.68 \\
\hline Preocupación salud & 7.54 & 3.34 & 6.60 & 2.93 & 5.29 & 2.40 & 2.06 \\
\hline Salud Mental Total & 64.46 & 20.28 & 56.74 & 20.51 & 31.94 & 5.89 & $8.56^{* * *}$ \\
\hline $\begin{array}{l}\text { Calidad de vida en } \\
\text { general }\end{array}$ & 12.48 & 3.03 & 10.56 & 2.93 & 6.93 & 3.25 & $11.86^{* * *}$ \\
\hline Bienestar emocional & 17.40 & 5.03 & 17.64 & 4.85 & 13.77 & 3.53 & 2.33 \\
\hline $\begin{array}{l}\text { Limitaciones probl. } \\
\text { emocionales }\end{array}$ & 14.96 & 10.68 & 10.88 & 11.36 & .00 & .00 & $6.21^{*}$ \\
\hline Función cognitiva & 10.01 & 3.83 & 9.25 & 3.88 & 4.50 & 1.60 & $6.93^{* *}$ \\
\hline
\end{tabular}

${ }^{*} p<.05 ;{ }^{* *} p<.01 ;{ }^{* *} p<.001$

Y, por último, el tiempo de enfermedad estuvo relacionado inversamente con la salud física, y el grado de discapacidad presentó relaciones significativas con todas las medidas de salud física, a excepción de la escala de Dolor, y de magnitud más baja para las medidas de salud mental (tabla 7 ).

Tabla 7. Correlaciones de Pearson entre las escalas y subescalas del MSQOL-54 y variables tiempo de enfermedad y grado de discapacidad en mujeres y hombres con EM.

\begin{tabular}{|c|c|c|c|c|}
\hline \multirow[t]{2}{*}{ MSQOL-54 } & \multicolumn{2}{|c|}{$\begin{array}{l}\text { Tiempo de } \\
\text { enfermedad }\end{array}$} & \multicolumn{2}{|c|}{$\begin{array}{c}\text { Grado de } \\
\text { discapacidad }\end{array}$} \\
\hline & $\begin{array}{l}\text { Mujeres } \\
(n=84)\end{array}$ & $\begin{array}{l}\text { Hombres }(n= \\
31)\end{array}$ & $\begin{array}{l}\text { Mujeres } \\
(n=84)\end{array}$ & $\begin{array}{l}\text { Hombres } \\
(n=31)\end{array}$ \\
\hline Salud Física Total & -.254 & -.017 & $-.585^{\star * *}$ & $-.443^{* * *}$ \\
\hline Salud física & $-.452^{*}$ & -.216 & $-.690^{\star * *}$ & $-.697^{* \star *}$ \\
\hline Percepción salud & -.182 & .074 & $-.446^{* *}$ & $-.280^{*}$ \\
\hline Energía & -.011 & -.076 & -.307 & -.200 \\
\hline Limitaciones físicas & -.328 & -.102 & $-.549 * *$ & $-.482^{* * *}$ \\
\hline
\end{tabular}




\begin{tabular}{lllll}
\hline \multicolumn{1}{c}{ MSQOL-54 } & \multicolumn{2}{c}{$\begin{array}{c}\text { Tiempo de } \\
\text { enfermedad }\end{array}$} & \multicolumn{2}{c}{$\begin{array}{c}\text { Grado de } \\
\text { discapacidad }\end{array}$} \\
\hline & $\begin{array}{l}\text { Mujeres } \\
(\mathbf{n = 8 4})\end{array}$ & $\begin{array}{l}\text { Hombres } \mathbf{( n =} \\
\mathbf{3 1})\end{array}$ & $\begin{array}{l}\text { Mujeres } \\
(\mathbf{n = 8 4})\end{array}$ & $\begin{array}{l}\text { Hombres } \\
(\mathbf{n = 3 1})\end{array}$ \\
\hline Dolor & .131 & -.091 & -.007 & -.192 \\
Función sexual & -.212 & -.032 & $-.492^{* *}$ & $-.254^{*}$ \\
Función social & .124 & .001 & -.235 & -.218 \\
Preocupación salud & -.173 & .160 & $-.482^{* *}$ & -.113 \\
Salud Mental Total & .140 & .093 & -.156 & $-.291^{*}$ \\
Calidad de vida general & -.033 & -.055 & $-.429^{*}$ & $-.370^{* *}$ \\
Bienestar emocional & .346 & .163 & .037 & .036 \\
Limitaciones probl. & .176 & .052 & .015 & $-.386^{* * *}$ \\
emocionales & & .053 & -.083 & -.116 \\
$\begin{array}{l}\text { Función cognitiva } \\
{ }^{*} p<.05 ;{ }^{* *} p<.01 ;{ }^{* * *} p<.001\end{array}$ & -.026 & & &
\end{tabular}

Respecto a la evaluación de las personas cuidadoras, las medidas de salud de las personas cuidadoras, la puntuación media de la muestra total en el GHQ-12 fue de 12.89 ( $D T=5.49)$. Los resultados mostraron diferencias significativas en el GHQ-12 en función del sexo de la persona cuidadora principal $[t(77)=2.714, p=.008]$, siendo superior la media de las mujeres (Media $=14.29, D T=5.88$ ) a la de los hombres (Media $=11.03, D T=4.35)$, es decir, las mujeres manifestaron peor salud mental que los hombres. Las puntuaciones, totales y desagregadas por sexo, son significativamente superiores a los datos normativos de la adaptación española del cuestionario $^{(14)}$, obteniendo diferencias significativas tanto en la puntuación total $[t(78)$ $=7.06, p=.000]$, como en la de los hombres $[t(33)=4.94, p=.000]$ y las mujeres $[t$ $(44)=5.68, p=.000]$.

Por lo que respecta a la salud física autopercibida, un $48,7 \%$ de la muestra valoró su salud como buena, un $28,2 \%$ regular, un $15,4 \%$ muy buena, un $5,1 \%$ mala y un $2,6 \%$ como muy mala. El nivel de salud autopercibida con una valoración positiva en la Encuesta Nacional de Salud ${ }^{15}$ es de un $80 \%$, mientras que en nuestra muestra de personas cuidadoras de un $64 \%$. Las mujeres cuidadoras afirmaron tener peor salud que las mujeres de la población general. Solo un $59.1 \%$ obtuvo una salud autopercibida buena, frente al $71.3 \%$ de las mujeres de la población general española. Los resultados para los hombres cuidadores fueron similares, un $76.5 \%$, frente al $79.4 \%$ de los hombres de la población general española.

En cuanto a las enfermedades que padecían o habían padecido, las dolencias más frecuentes fueron el dolor de espalda crónico lumbar (36.4\%) y el dolor de espalda cervical (35.1\%). Otras enfermedades relativamente frecuentes fueron depresión, ansiedad u otros trastornos mentales (26.9\%), hemorroides $(23.1 \%)$ y migrañas o dolores de cabeza frecuentes $(21.8 \%)$.

En la tabla 8 se muestran los resultados de las correlaciones entre variables de salud y las subescalas del MSQOL-54 de las personas con EM y las variables de salud de las mujeres y hombres cuidadores. 
Tabla 8. Correlaciones de Pearson entre las escalas y subescalas del MSQOL-54 y variables de salud de la persona cuidadora

\begin{tabular}{|c|c|c|c|c|c|c|}
\hline & \multicolumn{3}{|c|}{ Mujeres cuidadoras } & \multicolumn{3}{|c|}{ Hombres cuidadores } \\
\hline $\begin{array}{c}\text { Personas EM } \\
\text { MSQOL-54 }\end{array}$ & GHQ-12 & AP & ENF & $\begin{array}{c}\text { GHQ- } \\
12\end{array}$ & AP & ENF \\
\hline Tiempo de enfermedad &,$- 354^{*}$ &,- 122 &,- 070 & ,006 & ,174 & 119 \\
\hline Grado de discapacidad &,- 078 & , 180 &, $368^{*}$ &,- 156 & , 189 & ,246 \\
\hline Salud Física Total &,- 239 &,- 287 &,- 243 & ,090 &,- 013 &,- 085 \\
\hline Salud física &,- 091 &,- 195 &,- 205 & 012 &,- 182 &,- 337 \\
\hline Percepción salud &,- 221 &,- 252 &,- 084 & 022 & ,019 &,- 185 \\
\hline Energía &,$- 301^{*}$ &,- 266 &,- 045 & 022 & ,112 & ,069 \\
\hline Limitaciones físicas &,- 264 &,$- 353^{*}$ &,$- 382^{* *}$ &,- 006 & ,003 &,- 274 \\
\hline Dolor &,- 169 &,- 197 &,- 162 & ,218 &,- 080 & ,289 \\
\hline Función sexual &,- 236 &,- 251 &,- 256 & ,348* &,- 198 & , 171 \\
\hline Función social &,$- 380^{*}$ &,$- 300^{*}$ &,- 164 & 148 & 113 & 075 \\
\hline Preocupación salud &,- 221 &,- 197 &,- 206 &,- 095 & ,227 & ,092 \\
\hline Salud Mental Total &,$- 409^{* *}$ &,$- 326^{*}$ &,- 199 &,- 038 &,- 066 & ,024 \\
\hline Calidad de vida general &,$- 334^{*}$ &,- 318 &,- 188 &, 000 &,- 041 &,- 036 \\
\hline Bienestar emocional &,$- 403^{* *}$ &,- 228 &,- 073 &,- 039 &,- 123 & ,077 \\
\hline $\begin{array}{l}\text { Limitaciones probl } \\
\text { emocionales }\end{array}$ &,$- 372^{*}$ &,$- 325^{*}$ &,- 189 &,- 072 &,- 187 &,- 105 \\
\hline Función cognitiva &,- 179 &,- 127 & -112 & 134 & 103 & 239 \\
\hline
\end{tabular}

${ }^{\star} p<.05^{* \star} p<.01 ; \mathrm{AP}=$ Autopercepción de Salud; ENF $=\mathrm{N}^{\circ}$ enfermedades

En las mujeres cuidadoras, los resultados mostraron relaciones significativas inversas entre el malestar psicológico de las cuidadoras con: el tiempo de enfermedad, las subescalas de energía y función social, la Salud Mental Total, y las subescalas de calidad de vida general, bienestar emocional y limitaciones por problemas emocionales. Es decir, a mayor malestar psicológico de las cuidadoras, menor CVRS Física en las escalas de Energía y Función Social, y menor calidad de vida en Salud Mental Total y en las subescalas de Calidad de vida general, Bienestar emocional y Limitaciones por problemas emocionales. La autopercepción de salud en las mujeres cuidadoras también se relacionó inversamente con limitaciones físicas, con Salud Mental Total y con Limitaciones por problemas emocionales, de tal manera que la mejor autopercepción de salud en las mujeres cuidadoras, mayor calidad de vida de las personas con EM en estas subescalas. El número de enfermedades en las mujeres cuidadoras estuvo directamente relacionado con el grado de discapacidad, e inversamente con la calidad de vida en la escala de Limitaciones físicas. En los hombres cuidadores solo se encontraron relaciones directas entre el malestar psicológico del cuidador y la calidad de vida en la subescala de Función sexual.

\section{DISCUSIÓN}

Los resultados obtenidos confirman que la CVRS evaluada a través del MSQOL-54, es menor en las personas con EM que en la población general. Según los datos de la Encuesta Nacional de Salud 2011/12, en relación a la Calidad de vida relacionada con la Salud de la población adulta española (15), la puntuación media de CVRS para los rangos de edad de 35 a 44 años es de 81.4 sobre 100 puntos y la media para los rangos de 45 a 54 años es de 77.2 sobre 100 puntos. En ambos rangos, la CVRS es igualmente más alta que las obtenidas para Salud Física y Mental en nuestra muestra. Estos resultados concuerdan con los resultados obtenidos por el estudio de Aymerich 
et al. (8) sobre la CVRS de los pacientes con EM en el que, de acuerdo con las ocho dimensiones del SF-36, ésta fue inferior en todas las dimensiones en comparación con los datos normativos para la población en general.

Nuestros resultados también confirman que la CVRS de las personas con EM, evaluada a través del MSQOL-54, se muestra afectada a nivel general, siendo ligeramente mayor la afectación de la CVRS física que la mental, estando relacionadas principalmente a las limitaciones físicas asociadas a la enfermedad y a la función cognitiva respectivamente, no existiendo diferencias significativas entre los hombres y mujeres de la muestra. Con respecto a la Salud Física, los resultados muestran que la enfermedad provoca limitaciones físicas para la realización de esfuerzos intensos, como correr, subir escaleras, hacer grandes recorridos andando, etc., y menos limitaciones en la realización de aquellas actividades que hacen referencia a su autonomía como bañarse o vestirse. En otros estudios se ha señalado que en estadios tempranos de la enfermedad las dimensiones más afectadas son el dolor corporal y la vitalidad, así como que a medida que progresa la enfermedad se afectan más aquellas dimensiones del cuestionario SF-36 relacionadas con la función física(17). De nuevo encontramos similitud con nuestros hallazgos, en los que los pacientes se encuentran en un estadio temprano y la presencia de dolor es considerable, provocando limitaciones para la realización de actividades físicas cotidianas, así como dentro de la función social para la realización de una actividad laboral normalizada. En este sentido, Grasso et al. ${ }^{(18)}$ consideran que las principales repercusiones que tiene el dolor consisten en la reducción de la vitalidad, el empeoramiento de la función física y el deterioro de la salud mental. En relación a la función cognitiva, según el estudio realizado por Amato, Ponziani, Siracusa y Sorbi(19), su afectación incide principalmente en la velocidad de procesamiento de la información y la memoria episódica y de trabajo. Esta afectación puede repercutir notoriamente en las actividades de la vida diaria, implicando una mayor dependencia para el cuidado personal, colaboración para el desarrollo de las tareas de hogar, reducción de la participación en las actividades sociales e incremento de las dificultades asociadas al ámbito laboral.

Coincidiendo con los resultados del estudio de Aymerich et al. ${ }^{(8)}$, no se encuentran diferencias en las dimensiones CVRS entre hombres y mujeres. Sin embargo, al igual que el estudio realizado por Alarcón et al. (20), se confirma el peso que variables sociodemográficas como la edad, escolaridad, ocupación y estado civil tienen sobre la CVRS. La edad es un factor determinante en la CVRS de las personas con EM, siendo las más jóvenes, entre 20 y 30 años, las que presentan mejor CVRS. Asimismo, la situación laboral, el nivel educativo y el estado civil también mostraron relación con la CVRS del paciente con EM. Las personas con EM que trabajan y las que tienen un nivel educativo superior tienen una mejor CVRS en prácticamente todas las dimensiones de Salud Física y Mental. Las personas solteras obtuvieron también una mayor CVRS Física y Mental que los casados o los separados.

Como era de esperar, a mayor duración de la enfermedad menor CVRS Física en los pacientes con EM. El grado de discapacidad, coincidiendo con estudios similares ${ }^{(21)}$, estuvo relacionado con menor CVRS Física y Mental en casi todas sus dimensiones, afectando más a la salud física y a la calidad de vida en general, y más débilmente a la percepción de la salud, a la función social, a la energía y a las limitaciones por problemas emocionales. 
Por lo que respecta a la salud de la persona cuidadora principal, los resultados hallados indican que ésta es menor que la de la población general, tanto respecto a la salud física autopercibida como a la salud mental. Estos resultados están en la línea de investigaciones previas sobre variables de salud y adaptación psicológica en personas cuidadoras ${ }^{(22-24)}$. Del mismo modo, estos resultados son coincidentes con estudios llevados a cabo con personas cuidadoras de pacientes con EM que indican que la calidad de vida de éstos es menor que la de la población general( $6,8,25,26)$, así como que presentan una mayor prevalencia de síntomas ansioso-depresivos ${ }^{(8,25)}$. Asimismo, la peor salud de las mujeres cuidadoras con respecto a la salud de los hombres cuidadores también ha sido señalada en estudios previos ${ }^{(27-29)}$. Nuestros resultados vienen a confirmar estas diferencias en una muestra específica de personas cuidadoras, subrayando la importancia de la inclusión de la perspectiva de género en la investigación sobre la salud de las personas cuidadoras de pacientes con EM.

Respecto a la relación entre la CVRS de la persona con EM y la salud de las personas cuidadoras, los resultados del estudio permiten confirmar esta relación y señalar distintas asociaciones significativas. La afectación de la salud física de la persona con EM y las limitaciones en su vida diaria a causa de la enfermedad se asocia con un mayor número de enfermedades en la persona que lo cuida (especialmente dolor de espalda lumbar y cervical, hemorroides, migrañas o dolores de cabeza frecuentes, depresión y ansiedad), del mismo modo que el grado de limitaciones por problemas emocionales y relacionados con la función sexual del paciente con una peor autopercepción de salud en la persona cuidadora. Por otra parte, un menor grado de bienestar emocional, salud mental y calidad de vida en general en la persona con EM se asocia a una mayor peor salud mental en las personas cuidadoras.

Los resultados encontrados ofrecen nuevos datos que vienen a complementar investigaciones previas sobre el tema que han señalado la importancia de la relación entre la severidad y el deterioro físico de la persona con EM y la salud del cuidador/a $(30,31)$, considerando que los tratamientos que retrasan la pérdida de función de la persona con EM no sólo suponen un factor esencial para su CVRS sino que, además, cobran una relevante importancia al implicar un beneficio añadido para la salud de las personas cuidadoras.

Entre las limitaciones del estudio pueden incluirse las características de la muestra que, aunque incluyó a personas de ambos sexos y sujetos provenientes tanto del ámbito hospitalario como abierto, fundamentalmente estuvo compuesta de personas en estadios tempranos de la enfermedad, lo que limita la generalización de los resultados encontrados. Del mismo modo, los participantes fueron seleccionados utilizando muestreo por selección intencionada o muestreo de conveniencia, siendo invitados a participar de forma voluntaria en el estudio, razón por la cual el número de cuidadores fue menor que el de las personas con EM.

\section{CONCLUSIONES}

Los resultados obtenidos señalan la significativa afectación de la CVRS de las personas con EM y su relación directa con la salud de las personas cuidadoras principales. Los participantes de nuestra muestra tienen una CVRS Física moderada 
siendo el área de las limitaciones físicas que les produce la enfermedad, la más afectada. Respecto a la CVRS Mental, se destaca la mayor afectación de la subescala relacionada con la Función cognitiva. La edad, la situación laboral, el nivel educativo y el estado civil son factores determinantes en la CVRS de la persona con EM.

Respecto a la relación entre la CVRS de la persona con EM y la salud de las personas cuidadoras, los resultados del estudio permiten confirmar la relación entre las limitaciones físicas de la persona con EM y mayor número de enfermedades en los cuidadores, y la relación entre las limitaciones por problemas emocionales y problemas en la función sexual, y la peor autopercepción de salud en el cuidador.

\section{REFERENCIAS}

1. Stenager, E.N., Stenager E., Koch-Henricksen, N., Bronnum-Hansen, H., Hyllested, K., Jensen K.,...Bille-Brahe, U. (1992). Suicide and multiple sclerosis: an epidemiological investigation. Journal of Neurology, Neurosurgery \& Psychiatry, 55(7), 542-545. http://dx.doi.org/10.1136/jnnp.55.7.542

2. Mitchell, A.J., Benito-León, J., González, J.M., \& Rivera-Navarro, J. (2005). Quality of life and its assessment in multiple sclerosis: integrating physical and psychological components of wellbeing. The Lancet Neurology, 4(9), 556-566. http://dx.doi.org/10.1016/s1474-4422(05)70166-6

3. Schwartzman, L. (2003). Calidad de vida relacionada con la salud: Aspectos conceptuales. Ciencia y Enfermería, 9(2), 9-21.http://dx.doi.org/10.4067/s071795532003000200002

4. Testa, M., \& Simonson, D.C. (1996). Assessment of Quality-of-Life Outcomes. New England Journal of Medicine, 334(13), 835-840. http://dx.doi.org/10.1056/nejm199603283341306

5. Hernández, M.A. (2000). Tratamiento de la esclerosis múltiple y calidad de vida. Revista de Neurología, 30(12), 1242-1245. https://www.neurologia.com/articulo/99517

6. Rivera-Navarro, J., Morales-González, J.M., Benito-León, J., \& Mitchell, A.J. (2008). Dimensión social y familiar: experiencias de cuidadores y personas con esclerosis múltiple. El estudio GEDMA. Revista de Neurología, 47(6), 281-285. https://www.neurologia.com/articulo/2008071

7. Olascoaga, J. (2010). Calidad de vida y esclerosis múltiple. Revista de Neurología, 51(5), 279-288. https://www.neurologia.com/articulo/2010277

8. Aymerich, M., Guillamón, I., \& Jovell, A. J. (2009). Health-related quality of life assessment in people with multiple sclerosis and their family caregivers. A multicenter study in Catalonia (Southern Europe). Patient Prefer Adherence, 3, 311-321.http://dx.doi.org/10.2147/ppa.s6217

9. McKeown, L.P., Porter-Armstrong, A.P., \& Baxter, G.D. (2003). The needs and experiences of caregivers of individuals with multiple sclerosis: a systematic review. Clinical Rehabilitation, 17(3), 234-248. http://dx.doi.org/10.1191/0269215503cr618oa

10. Carreón-Guarnizo, E., Andreu-Reinon, E., Cerdán-Sánchez, M., Carrasco-Torres R., Hernández-Clares, R., Prieto-Valiente L.,...Meca-Lallana, J.E. (2016). Prevalencia de la Esclerosis Múltiple en la Región de Murcia [Prevalence of multiple sclerosis in the Region of Murcia]. Revista de Neurología, 62(9), 396-402.

11. Vickrey, B.G., Hays, R.D., Harooni, R., Myers, L.W., \& Ellison, G.W. (1995). A 
health-related quality of life measure for multiple sclerosis. Quality of Life Research, 4, 187-206. http://dx.doi.org/10.1007/bf02260859

12. Aymerich, M., Guillamón, I., Perkal, H., Nos, C., Porcel, J., Berra, S.,...Montalbán, X. (2006). Adaptación al español del cuestionario específico MSQOL-54 para pacientes con esclerosis múltiple. Neurología, 21(4), 181-187.

13. Goldberg, D., \& Williams, P. (1988). A user's guide to the General Health Questionnaire. Windsor, UK: NFER-Nelson.

14. Sánchez-López, M.P., \& Dresch, V. (2008). The 12-item General Health Questionnaires (GHQ-12): reliability, external validity and factor structure in the Spanish population. Psicothema, 20(4), 839-843.

15. Instituto Nacional de Estadística, INE. (2013). Encuesta Nacional de Salud 20112012. Ministerio de Sanidad, Servicios Sociales e Igualdad. Gobierno de España.

16. Sánchez-López, M.P., \& Limiñana-Gras, R.M. (2017). Health from a Gender Perspective. In M.P. Sánchez-López \& R.M. Limiñana-Gras (Eds.), The Psychology of Gender and Health: Conceptual and Applied Global Concerns (pp.152). Elseiver/Academic Press. http://dx.doi.org/10.1016/b978-0-12-8038642.00001-8

17. Delgado-Mendilívar, J.M., Cadenas-Díaz, J.C., Fernández-Torrico, J.M., NavarroMascarell, G., \& Izquierdo, G. (2005). Estudio de la calidad de vida en la Esclerosis Múltiple. Revista de Neurología, 41(5), 257-262.

18. Grasso, M.G., Clemenzi, A., Tonini, A., Pace, L., Casillo, P., Cuccaro, A.,...Troisi, E. (2008). Pain in multiple sclerosis: a clinical and instrumental approach. Multiple Sclerosis, 14(4), 506-513. http://dx.doi.org/10.1177/1352458507085553

19. Amato, M.P., Ponziani, G., Siracusa, G., \& Sorbi, S. (2001). Cognitive dysfunction in early-onset multiple sclerosis: a reappraisal after 10 years. Archives of Neurology, 58(10), 1602-1606. http://dx.doi.org/10.1001/archneur.58.10.1602

20. Alarcón, G. S., McGwin, G., Uribe, A., Friedman, A. W., Roseman, J. M.,... Fessler, B. J. (2004). Systemic lupus erythematosus in a multiethnic lupus cohort (LUMINA). XVII. Predictors of self-reported health-related quality of life early in the disease course. Arthritis Care \& Research, 51(3), 465-474. http://dx.doi.org/10.1002/art.20409

21. Hopman, W. M., Coo, H., Edgar, C. M., McBride, E. V., Day, A. G., \& Brunet, D. G. (2007). Factors Associated with Health-Related Quality of Life in Multiple Sclerosis. The Canadian Journal of Neurological Sciences, 34(02), 160-166. http://dx.doi.org/10.1017/s0317167100005989

22. Cuéllar-Flores, I., \& Sánchez-López, M. P. (2012). Adaptación psicológica en personas cuidadoras de familiares dependientes. Clínica y Salud, 23(2), 141-152. http://dx.doi.org/10.5093/cl2012a9

23. De Andrés-Jiménez, Elena y Limiñana-Gras, Rosa María (2014). El afrontamiento del cuidado en familiares cuidadores de personas con demencia: dimensiones cognitivas y el manejo de la ira. Revista Iberoamericana de Diagnóstico y Evaluación - e Avaliação Psicológica [Internet]. 2014;1(37),169-185.

24. Manso-Martínez, M. E., Sánchez-López, M. P., \& Cuéllar-Flores, I. (2013). Salud y sobrecarga percibida en personas cuidadoras familiares de una zona rural. Clínica y Salud, 24(1), 37-45. http://dx.doi.org/10.5093/cl2013a5

25. Figved, N., Myhr, K.M., Larsen, J.P., \& Aarsland, D. (2007) Caregiver burden in multiple sclerosis: the impact of neuropsychiatric symptoms. Journal of Neurology, Neurosurgery and Psychiatry, 78(10), 1097-1102. http://dx.doi.org/10.1136/jnnp.2006.104216

26. Patti, F., Pozzilli, C., Montanari, E., Pappalardo, A., Piazza, L., Levi, A.,...Pesci, I. (2007). Effects of education level and employment status on HRQoL in early 
relapsing-remitting multiple sclerosis. Multiple Sclerosis, 13(6), 783-791.

http://dx.doi.org/10.1177/1352458506073511

27. Cuéllar-Flores, I., Sánchez-López, M. P., Limiñana-Gras, R. M., \& Colodro-Conde, L. (2014). The GHQ-12 for the Assessment of Psychological Distress of Family Caregivers. Behavioral Medicine, 40(2), 65-70. http://dx.doi.org/10.1080/08964289.2013.847815

28. Pinquart, M., \& Sörensen, S. (2006). Gender differences in caregiver stressors, social resources, and heath: an update meta-analysis. Journal of Gerontology, Series B: Psychological Sciences, 61(1), 33-45. http://dx.doi.org/10.1093/geronb/61.1.p33

29. Yee, J., \& Schulz, R. (2000). Gender differences in psychiatric morbidity among family caregivers: a review and analysis. The Gerontologist, 40(2), 147-164. http://dx.doi.org/10.1093/geront/40.2.147

30. Acaster, S., Perard, R., Chauhan, D., \& Lloyd, A.J. (2013). A forgotten aspect of the NICE reference case: an observational study of the health related quality of life impact on caregivers of people with multiple sclerosis. BMC Health Services Research, 13(1), 346. http://dx.doi.org/10.1186/1472-6963-13-346

31. Forbes, A., While, A., \& Mathes, L. (2007). Informal carer activities, carer burden and health status in multiple sclerosis. Clinical Rehabilitation, 21(6), 563-575. http://dx.doi.org/10.1177/0269215507075035 